\title{
USP39 promotes ovarian cancer malignant phenotypes and carboplatin chemoresistance
}

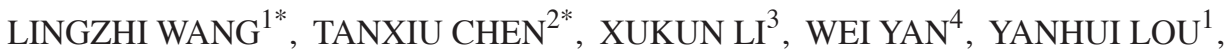 \\ ZHIHUA LIU ${ }^{3}$, HONGYAN CHEN ${ }^{3}$ and ZHUMEI CUI ${ }^{1}$ \\ ${ }^{1}$ Department of Obstetrics and Gynecology, The Affiliated Hospital of Qingdao University, Qingdao, Shandong 266061; \\ ${ }^{2}$ Department of Science and Education, Jiangxi Key Laboratory of Translational Cancer Research, \\ Jiangxi Cancer Hospital, Nanchang, Jiangxi 330029; ${ }^{3}$ State Key Laboratory of Molecular Oncology, \\ National Cancer Center/Cancer Hospital, Chinese Academy of Medical Sciences and Peking Union Medical College, \\ Beiing 100021; ${ }^{4} 6$ th Department of Internal Medicine, Jiangxi Cancer Hospital, Nanchang, Jiangxi 330029, P.R. China
}

Received October 19, 2018; Accepted May 24, 2019

DOI: $10.3892 /$ ijo.2019.4818

\begin{abstract}
Ubiquitin-specific protease 39 (USP39), as one of the deubiquitinating enzymes (DUBs), exhibits aberrant an expression and has oncogenic functions in several types of cancer. However, the function and underlying molecular mechanisms of action of USP39 in ovarian cancer remain largely undetermined. The present study thus aimed to investigate whether USP39 is a promising tumor-associated gene and whether it could be a viable target for overcoming chemotherapeutic resistance in ovarian cancer. The present study identified that USP39 was highly expressed in ovarian cancer samples with carboplatin resistance. A series of functional assays revealed that the knockdown of USP39 in ES2 and SKOV3 cells significantly decreased cell proliferation, induced cell cycle arrest at the $\mathrm{G} 2 / \mathrm{M}$ phase and impaired the cell colony formation ability. USP39 deficiency enhanced the carboplatin-induced apoptosis of the SKOV3 cells via the activation of poly-ADP ribose polymerase and caspase-3. USP39 knockdown led to the inhibition of cell migration and invasion. The opposite effects were observed when USP39 was overexpressed in the ES2 and SKOV3 cells. In vivo animal models revealed that the
\end{abstract}

Correspondence to: Professor Hongyan Chen, State Key Laboratory of Molecular Oncology, National Cancer Center/Cancer Hospital, Chinese Academy of Medical Sciences and Peking Union Medical College, 17 Panjiayuan Nanli, Chaoyang, Beijing 100021, P.R. China

E-mail: chenhongyan@cicams.ac.cn

Professor Zhumei Cui, Department of Obstetrics and Gynecology, The Affiliated Hospital of Qingdao University, Qingdao, 16 Jiangsu Road, Shandong 266061, P.R. China

E-mail: cuizhumei1966@126.com

*Contributed equally

Key words: ubiquitin-specific protease 39, ovarian cancer, proliferation, apoptosis, therapy resistance subcutaneous transplantation and intraperitoneal injection of USP39-overexpressing ES2 cells increased tumor burden with or without treatment with carboplatin. However, the knockdown of USP39 suppressed SKOV3 cell growth in vivo. Mechanistic analyses also demonstrated that USP39 induced the phosphorylation of extracellular signal-regulated kinase and AKT and increased the expression of epidermal growth factor receptor and cyclin B1. Collectively, the findings of this study suggest that USP39 may paly a vital role in regulating ovarian cancer malignant phenotypes and carboplatin resistance. Therefore, USP39 may prove to be a promising therapeutic target for patients with ovarian cancer.

\section{Introduction}

With a 5-year survival rate of $<25 \%$, ovarian cancer represents one of the most aggressive and challenging types of cancer as regards treatment. Ovarian cancer is the leading cause of cancer-associated mortality among all gynecological tumors, and the majority of patients with this type of cancer are diagnosed at advanced and metastatic stages $(1,2)$. Despite advances in systemic chemotherapies, including cisplatin and carboplatin, which are widely used at present, the prognosis of patients with ovarian cancer remains remarkably poor due to high incidences of chemoresistance $(1,3)$. To date, multiple molecular mechanisms involved in ovarian tumorigenesis and chemoresistance have been proposed; however, no reliable biomarkers have been identified as predictors of an individual's chemotherapeutic response $(1,4)$. Thus, the identification of leading factors mediating chemoresistance and the elucidation of the underlying mechanisms is of utmost importance, in order to develop novel and effective therapeutic strategies for preventing or reversing therapeutic resistance.

Ubiquitin-specific peptidase 39 (USP39) encodes a $65 \mathrm{kDa}$ SR-associated protein, which has been demonstrated to be involved in RNA splicing as a component of the U4/U6.U5 tri-small nuclear ribonucleoprotein (snRNP) $(5,6)$. Zebrafish USP39 displays the ability to recruit the tri-snRNPs to the pre-spliceosome and target retinoblastoma (rb1) and E2F transcription factor 4 (e2f4) expression to regulate embryonic 
pituitary homeostasis (7). In accordance with its previous reported role in messenger RNA (mRNA) processing, USP39 has also been implicated as a key factor in regulating the splicing of Aurora B and other mRNAs to maintain mitotic spindle checkpoint integrity (8). Notably, despite being classified as a member of the deubiquitinating enzymes (DUBs), USP39 is fully deprived of protease activity (9). Recent evidence suggests that USP39 plays a vital role in regulating the malignant phenotypes of various cancer types $(1,5)$. For instance, the overexpression of USP39 promotes tumorigenesis in prostate cancer; mechanistic investigations have demonstrated that the silencing of USP39 downregulates epidermal growth factor receptor (EGFR) expression by inducing a splicing defect via the retention of intron 2 between exons 2 and 3, thus affecting the expression of the $3^{\prime}$ end of EGFR (10). The silencing of USP39 has been shown to inhibit cell growth and colony formation of breast cancer in vitro (11). Additionally, USP39 has been identified as an indispensable gene for the survival of KRAS-dependent lung cancer and colorectal carcinoma (8). However, little is known about the biological functions and the role of USP39 in the chemosensitivity of human ovarian cancer.

The present study thus aimed to investigate the roles of USP39 in ovarian cancer. USP39 was found to be highly expressed in carboplatin-resistant ovarian cancer tissues compared with carboplatin-sensitive tissues. Functional assays demonstrated that USP39 plays a crucial role in regulating malignant phenotypes and chemoresistance in ovarian cancer cells. In addition, USP39 confers ovarian cancer cell chemoresistance via the AKT/extracellular signal-regulated kinase (ERK) signaling pathway. Therefore, the findings of this study suggest that USP39 may be a potential molecular target for ovarian cancer therapy, and may provide novel insight into the progression and chemotherapeutic resistance of ovarian cancer.

\section{Materials and methods}

Clinical specimens. In the present study, a total of 119 clinical specimens from patients with ovarian cancer who underwent initial surgical treatment at the Department of Gynecologic Oncology of the Chinese Academy of Medical Sciences Cancer Hospital (Beijing, China) were collected from May, 2007 to January, 2013. All patients provided informed consent to participate in the study. This study was approved by the Ethics Committee of the Chinese Academy of Medical Sciences Cancer Hospital.

Immunohistochemistry (IHC). IHC was performed as previously described (12). USP39 expression was assessed using a rabbit monoclonal antibody (ab131244; Abcam) at a 1:50 dilution at $4^{\circ} \mathrm{C}$ overnight, according to the manufacturer's instructions. As negative controls, sections incubated with rabbit immunoglobulin G (1:1000; ab6721; Abcam) instead of primary antibodies were used. Following incubation with horseradish peroxidase (HRP)-conjugated secondary antibodies (OriGene Technologies, Inc.) at room temperature for $1 \mathrm{~h}$, the positive signals were visualized with 3,3'-diaminobenzidine (OriGene Technologies, Inc.) as a substrate. All slides were scanned with an Aperio scanning system (Aperio Group, LLC) and Aperio Image Scope software (version 10.2.2.2317, Aperio Technologies) was employed for the quantitative analysis of
USP39 protein expression. For analysis, 4-6 different areas of the slide were randomly selected. The intensity score was graded with a score ranging from 0 to 3 according to the percentage of positively stained tumor cells. When $0-10 \%$ of tumor cells were stained, a score of 0 was given; when $10-25 \%$ of tumor cells were stained, a score of 1 was given; when $25-50 \%$ of tumor cells were stained, a score of 2 was given; and when $50-100 \%$ of tumor cells were stained, a score of 3 was given. Scores of 0 and 1 were considered to represent a low expression, while scores of 2 and 3 were considered to represent a high expression. The staining results of USP39 were evaluated according to the scoring criterion reported in a previous study (13).

Cell culture. The human ovarian cancer cell lines ES2, SKOV3 and the 293T cells were purchased from the American Tissue Culture Collection. The human ovarian cancer cell lines were cultured in RPMI-1640 (Gibco, Thermo Fisher Scientific) supplemented with $10 \%$ fetal bovine serum (FBS) (Gibco, Thermo Fisher Scientific), $100 \mathrm{U} / \mathrm{ml}$ ampicillin (Life Technologies, Gibco) and $100 \mu \mathrm{g} / \mathrm{ml}$ streptomycin (Life Technologies, Gibco). The $293 \mathrm{~T}$ cells were cultured in Dulbecco's modified Eagle medium (DMEM) supplemented with $10 \%$ FBS, $100 \mathrm{U} / \mathrm{ml}$ ampicillin and $100 \mu \mathrm{g} / \mathrm{ml}$ streptomycin. All cells were cultured at $37^{\circ} \mathrm{C}$ in $5 \% \mathrm{CO}_{2}$.

Construction of plasmids, lentivirus packaging and transfection. For the stable knockdown of USP39, two small hairpin RNA (shRNA) sequences (shUSP39\#1 and shUSP39\#2) targeting USP39 were respectively inserted into pSIH1-H1-Puro (Invitrogen, Thermo Fisher Scientific, Inc.), and sh-green fluorescence protein (GFP) (Invitrogen, Thermo Fisher Scientific, Inc.) was used as control. The two USP39 shRNA sequences were as follows: 5'-GTACTTTCAAGGCCG GGGT-3' (shUSP39\#1) and 5'-ACAAGCAGTACACA AGAAT-3' (shUSP39\#2). shRNA plasmids were transfected into the $293 \mathrm{~T}$ cells together with 3 packaging plasmids (3 $\mu \mathrm{g}$ PLP1, $3 \mu \mathrm{g}$ VSVG and $3 \mu \mathrm{g}$ PLP2) (Invitrogen, Thermo Fisher Scientific, Inc.) using Lipofectamine 2000 (Invitrogen; Thermo Fisher Scientific, Inc.) with OPTI-MEM (Thermo Fisher Scientific, Inc.). The virus-containing media were pooled at $48 \mathrm{~h}$ after transfection, centrifuged under $800 \mathrm{x}$ g for $10 \mathrm{~min}$ at $4^{\circ} \mathrm{C}$ and filtered by passing through a $0.45-\mu \mathrm{m}$ filtration device (EMD Millipore). Viral supernatant was added in a 1:3 dilution to previously seeded cell lines, supplemented with $5 \mu \mathrm{g} / \mathrm{ml}$ of polybrene (EMD Millipore). Stably transduced cells were selected with puromycin at a final concentration of $1 \mu \mathrm{g} / \mathrm{ml}$. For the stable overexpression of USP39, full-length complementary DNA of human USP39, which was generated by polymerase chain reaction (PCR) amplification, was cloned between the $\mathrm{XhoI}$ and $\mathrm{XbaI}$ sites of pLVX-IRES-Neo (Invitrogen, Thermo Fisher Scientific, Inc.) to prepare a constitutive lentiviral vector. A total of $8 \times 10^{6}$ $293 \mathrm{~T}$ cells were seeded in $100-\mathrm{cm}^{2}$ plates. pLVX-IRES-Neo or pLVX-IRES-Neo-USP39 lentiviral vectors $(7.5 \mu \mathrm{g})$ and the corresponding packaging plasmid $(6.5 \mu \mathrm{g} \mathrm{pCMV} \Delta 8.91,3.5 \mu \mathrm{g}$ VSVG and $2.5 \mu \mathrm{g}$ PLP2) were added to a mixture of Lipofectamine 2000 (Invitrogen; Thermo Fisher Scientific, Inc.) with OPTI-MEM (Thermo Fisher Scientific, Inc.). Following a similar transfection procedure as the one described 
above, stably transduced cells were selected with G418 (Sigma-Aldrich) at a final concentration of $400 \mu \mathrm{g} / \mathrm{ml}$. At $48 \mathrm{~h}$ following transfection, knockdown and overexpression efficiencies were evaluated by reverse transcription-quantitative PCR (RT-qPCR) and western blot analysis.

Cell proliferation assay. To quantify cell proliferation, $100 \mu \mathrm{l}$ stably transfected cells at a density of $1 \times 10^{4}$ cells $/ \mathrm{ml}(\mathrm{n}=6)$ were seeded in 96-well plates. A Cell Counting kit-8 (CCK-8) assay kit was used to detect cell viability at 5 time points. The cells were cultured in $100 \mu \mathrm{l} /$ well fresh medium mixed with CCK-8 solution (10:1) (Dojindo Molecular Technologies, Inc.) and incubated for $1 \mathrm{~h}$ at $37^{\circ} \mathrm{C}$. The optical density values were then measured at $450 \mathrm{~nm}$ using a spectrophotometer (Bio-Rad).

Apoptosis assay. The ES2 and SKOV3 cells were analyzed for apoptosis using an Annexin V-fluorescein isothiocyanate (FITC)/propidium iodide (PI) kit [Multisciences (Lianke) Biotech Co., Ltd.]. Briefly, the cells were seeded at $5 \times 10^{5}$ cells/well in 6 -well plates in triplicate. Following $24 \mathrm{~h}$ of incubation with carboplatin, the cells were harvested through trypsinization and washed twice with cold PBS. The cells were centrifuged at 3,000 $\mathrm{xg}$ at room temperature for $5 \mathrm{~min}$. The supernatant was then discarded and the pellet was resuspended in $500 \mu \mathrm{l} 1 \mathrm{X}$ binding buffer. Finally, the cells were incubated with $5 \mu 1$ FITC-conjugated Annexin V and $10 \mu \mathrm{l}$ of PI for $5 \mathrm{~min}$ at room temperature in the dark. The samples were analyzed by a FACSCalibur flow cytometer (BD Biosciences).

Analysis of the cell cycle. For cell cycle analysis, the cells were harvested and fixed with $70 \%$ ethanol for $18 \mathrm{~h}$ at $-20^{\circ} \mathrm{C}$ upon digestion with $0.05 \%$ trypsin. Upon washing with PBS and collecting the fixed cells by $800 \mathrm{x}$ g centrifugation at $4^{\circ} \mathrm{C}$ for $5 \mathrm{~min}$, samples were resuspended with $500 \mu \mathrm{l}$ DNA staining solution and then incubated in $37^{\circ} \mathrm{C}$ for $30 \mathrm{~min}$. Analysis was performed on a FACSCalibur flow cytometer (BD Biosciences).

RT-qPCR analysis. Total RNA was extracted using TRIzol reagent (Invitrogen; Thermo Fisher Scientific, Inc.). The cDNA was synthesized by the FastQuant RT kit [TIANGEN Biotech (Beijing) Co. Ltd.] using $100 \mathrm{ng}$ of total RNA. Quantitative analysis was performed using diluted cDNA combined with the SYBR-Green PCR Master Mix (Applied Biosystems; Thermo Fisher Scientific, Inc.), forward and reverse primers, and RNase-free water. The primer sequences were as follows: USP39 forward, 5'-GGTTTGAAGTCTCACGCCTAC-3' and reverse, 5'GGCAGTAAAACTTGAGGGTGT-3'; and $\beta$-actin forward, 5'-CCGTTCCGAAAGTTGC CTTTT-3' and reverse, 5'GAGGCGTACAGGGATAGCAC-3'. The RT-qPCR reactions were conducted using the following parameters: $95^{\circ} \mathrm{C}$ for $10 \mathrm{~min}, 40 \mathrm{cycles}$ at $95^{\circ} \mathrm{C}$ for $10 \mathrm{sec}$, at $60^{\circ} \mathrm{C}$ for $20 \mathrm{sec}$ and at $72^{\circ} \mathrm{C}$ for $30 \mathrm{sec}$. The relative mRNA expression in each sample was calculated using the $2^{-\Delta \Delta \mathrm{Cq}}$ method (14).

Western blot analysis. Total protein was extracted from whole cells using ice-cold cell lysis buffer (1\% Triton X-100; $10 \mathrm{mM}$ Tris-HCl, pH 7.4; 150 mM NaCl; 0.25\% sodium deoxycholate; 5 mM EDTA, pH 7.4) and assayed for protein concentration by a bicinchoninic acid assay (Pierce). Samples containing $15 \mu \mathrm{g}$ of protein were separated by $8-12 \%$ SDS-PAGE and transferred to polyvinylidene difluoride membranes (Merck KGaA). After blocking with $5 \%$ skimmed milk powder for $1 \mathrm{~h}$ at room temperature, the membranes were incubated with antibodies against USP39 (1:1,000; ab131244; Abcam), caspase-3 (1:1,000; \#9661; Cell Signaling Technology, Inc.), poly-ADP ribose polymerase (PARP) (1:1,000; \#9542; Cell Signaling Technology, Inc.) and $\beta$-actin (1:5,000; A5316; Sigma-Aldrich; Merck $\mathrm{KGaA}$ ) overnight at $4^{\circ} \mathrm{C}$, followed by incubation with a appropriate HRP-linked secondary antibody $(1: 2,000$; \#7074/\#7076; Cell Signaling Technology, Inc.) at room temperature for $1 \mathrm{~h}$. Samples were detected with an enhanced chemiluminescence detection system (Pierce; Thermo Fisher Scientific, Inc.).

Colony formation assay. For the colony formation assay, lentivirus-infected cells were seeded evenly in 6-well plates at an initial density of 2,000 cells/well and cultured in a $5 \% \mathrm{CO}_{2}$ incubator for 10 days at $37^{\circ} \mathrm{C}$. The cells were washed with ice-cold PBS, fixed with $4 \%$ paraformaldehyde (PFA), stained with crystal violet at room temperature for $30 \mathrm{~min}$ and rinsed 3 times with double distilled $\mathrm{H}_{2} \mathrm{O}$.

Wound healing assay. The cells were plated until they reached $90 \%$ confluence in 6-well plates. Cell monolayers were scratched with $200-\mu 1$ pipette tips. Upon forming wound gaps, the cells were washed with PBS twice to remove floating cells and cultured in serum-free medium. Cell migration into the wound area was observed under an inverted microscope (Leica Microsystems) at different time points. The speed of wound closure was analyzed by measuring the distance of the migrating cells from different areas for each wound.

Cell migration and invasion assays. For the cell migration assay, cells $\left(2 \times 10^{4}\right)$, which has been resuspended in $100 \mu 1$ serum-free medium, were added to the upper chambers of a Transwell plate (Corning Inc.). The lower chambers were filled with $600 \mu$ l RPMI-1640 complete medium containing $10 \%$ FBS. Following $24 \mathrm{~h}$ of incubation, the cells were fixed with $4 \%$ PFA for $30 \mathrm{~min}$ and stained with $0.1 \%$ crystal violet at room temperature for $1 \mathrm{~min}$. After washing gently with water, the cells were imaged and counted under a microscope (Leica Microsystems). For the cell invasion assay, Matrigel (Sigma-Aldrich; Merck KGaA) was diluted from $5 \mathrm{mg} / \mathrm{ml}$ to $1 \mathrm{mg} / \mathrm{ml}$ by serum free-cold RPMI-1640. The upper chambers of the Transwell plates were pre-coated with $100 \mu \mathrm{l}$ of the diluted Matrigel. The subsequent steps of the procedure were the same as those for the migration assay.

Subcutaneous xenograft models. Pathogen-free female BALB/c nude mice (age, 5-6 weeks; weight, 18-22 g, 6 mice per group) were provided standard laboratory chow and allowed free access to water, unless otherwise stated, in a climate-controlled room with a 12-h light/12-h dark cycle at the Animal Experiment Center of Cancer Hospital, Chinese Academy of Medical Sciences. Animal studies were approved by the Animal Care and Use Committee of Cancer Hospital, Chinese Academy of Medical Sciences. All procedures involving animals were conducted according to the guidelines for the care and use of laboratory animals. A total of $5 \times 10^{5}$ stably transfected ES2 cells were resuspended in $100 \mu 1$ normal saline and injected 


\section{Carboplatin-sensitive}
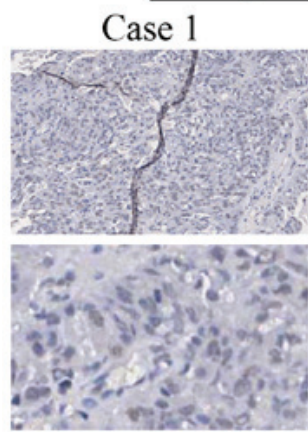

Case 2

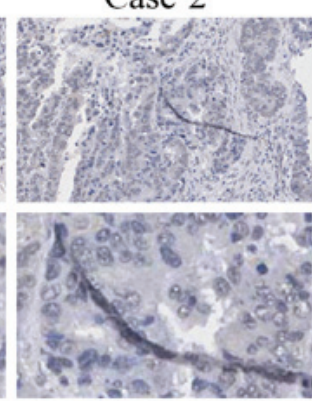

\section{Carboplatin-resistant}

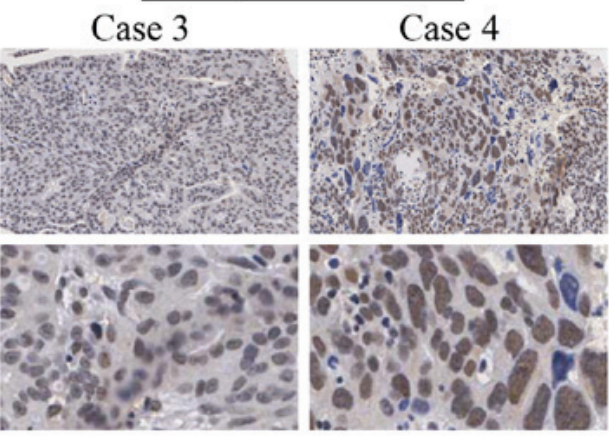

Figure 1. Characterization of USP39 expression in human carboplatin-sensitive (cases 1 and 2) and carboplatin-resistant (cases 3 and 4) ovarian cancer tissues by immunohistochemical analysis. Representative images are shown at x100 (upper panels) or x400 (lower panels) magnification. USP39, ubiquitin-specific protease 39 .

subcutaneously into the flank of each mouse with a $28 \mathrm{G}$ syringe. At 3 days post-inoculation, carboplatin was administered to the mice by intraperitoneal injection at a dose of $50 \mathrm{mg} / \mathrm{kg}$ every 3 days for 3 consecutive times as described previously (15). Tumors were measured using an electronic caliper twice per week, and tumor volume was calculated using the formula: Volume $=\left(\right.$ length $\mathrm{x}$ width $\left.{ }^{2}\right) / 2$. All mice were sacrificed by cervical dislocation at 21 days post-injection. Tumor samples were fixed in formalin for paraffin embedding and sectioned into $4 \mu \mathrm{m}$ slides for IHC analyses. The sections were incubated with antibodies against USP39 (1:50; ab131244; Abcam) and cleaved caspase-3 (1:50; \#9579; Cell Signaling Technology, Inc.) to measure the expression of USP39 and cleaved caspase-3.

Intraperitoneal tumorxenograftmodels.Luciferase-expressing control or USP39-overexpressing ES2 cells were cultured, harvested and suspended in RPMI-1640. Subsequently, $5 \times 10^{5}$ cells were injected intraperitoneally into the female BALB/c nude mice ( 3 mice per group). At 3 days post-inoculation, carboplatin was administered to the mice by intraperitoneal injection at a dose of $50 \mathrm{mg} / \mathrm{kg}$. Mice were housed for 10 days and injected with $200 \mu 115 \mathrm{mg} / \mathrm{ml}$ D-luciferin PBS solution 10 min prior to imaging. The whole animals were imaged using an IVIS Lumina XRMS In Vivo Imaging System (PerkinElmer, Inc.).

Statistical analysis. Statistical analyses were conducted using Prism software 6 (GraphPad Software, Inc.). The associations between USP39 expression and the patient clinicopathological data were assessed using a Chi-square test. All data are presented as the means \pm standard error of the mean of $\geq 3$ independent experiments. For 2-group comparison, analyses were performed with a Student's unpaired t-test (two-tailed). For multiple comparisons, analyses were performed with one-way ANOVA with Tukey's post hoc test. All experiments other than histological examinations were repeated $\geq 2$ times. $\mathrm{P}<0.05$ was considered to indicate a statistically significant difference.

\section{Results}

USP39 is overexpressed in ovarian cancer tissues and is associated with carboplatin resistance. USP39 expression was analyzed in carboplatin-sensitive and carboplatin-resistant human ovarian cancer tissues by IHC. The results revealed that USP39 was primarily expressed in the nucleus, but rarely in the cytoplasm (Fig. 1). In the carboplatin-resistant group, $60.71 \%$ of the specimens presented a strong positive expression vs. $41.27 \%$ in the carboplatin-sensitive group $(\mathrm{P}=0.034)$, determined by a Chi-square test (Table I). Subsequently, we analyzed whether the expression of USP39 can influence the clinicopathological characteristics of patients with ovarian cancer. As shown in Table I, USP39 expression was not associated with age, residual tumor size, level of cancer antigen 125 in primary tumors, International Federation of Gynecology and Obstetrics stage or tumor grade. These results indicate that high expression levels of USP39 in ovarian cancer tissues may be closely associated with the development of carboplatin resistance.

USP39 promotes cell proliferation and colony formation by inducing cell cycle phase arrest in ovarian cancer cells. To verify the role of USP39 in ovarian cancer cells, the ES2 and SKOV3 cell lines were transduced with shUSP39s or shGFP lentiviruses. The inhibitory effects of shUSP39s on its endogenous expression in the ES2 and SKOV3 cells were determined by RT-qPCR and western blot analysis (Fig. 2A and B). To examine the effects of USP39 on cell proliferation, a CCK-8 assay was performed. As shown in Fig. $2 \mathrm{C}$, the proliferative rate of the ES2 and SKOV3 cells was significantly lower in shUSP39s-transduced cells compared with the shGFP control cells. To further characterize the effects of USP39 on the cell proliferative capability, a colony formation assay was employed. As depicted in Fig. 2D, upon the knockdown of USP39, the capacity of colony formation of the ES2 and SKOV3 cells was substantially reduced. To elucidate the mechanisms through which USP39 modulates cell proliferation and colony formation, cell cycle distribution analysis was employed. The cell percentage in the G2/M phase was increased by USP39 knockdown in the SKOV3 cells, suggesting that cell growth suppression may be associated with increased $\mathrm{G} 2 / \mathrm{M}$ phase arrest (Fig. 2E). Cell cycle-associated protein analysis also revealed that the expression of cyclin B1 was suppressed following USP39 knockdown (Fig. 2F). The overexpression of USP39 in the ES2 cells indicated that USP39 significantly increased the cell proliferation and colony formation capacity of ES2 cells (Fig. 3). Taken together, these data support the important role of USP39 in regulating the proliferation of ovarian cancer cells. 
Table I. Association between the expression of USP39 and the patient clinicopathological features in the ovarian cancer tissue arrays.

\begin{tabular}{|c|c|c|c|c|}
\hline \multirow[b]{2}{*}{ Clinical variable } & \multirow[b]{2}{*}{ Patients } & \multicolumn{2}{|c|}{ USP39 expression [no. (\%)] } & \multirow[b]{2}{*}{$\mathrm{P}$-value } \\
\hline & & Low & High & \\
\hline All cases & 119 & $59(49.58)$ & $60(50.42)$ & \\
\hline Age at diagnosis (years) & $\begin{array}{l}\geq 60 \\
<60\end{array}$ & $\begin{array}{l}15(42.86) \\
44(52.38)\end{array}$ & $\begin{array}{l}20(57.14) \\
40(47.62)\end{array}$ & 0.344 \\
\hline Residual tumor size (cm) & $\begin{array}{l}\geq 2 \\
<2\end{array}$ & $\begin{array}{r}8(44.44) \\
51(50.50)\end{array}$ & $\begin{array}{l}10(55.56) \\
50(49.50)\end{array}$ & 0.636 \\
\hline $\begin{array}{l}\text { Level of CA125 in } \\
\text { primary tumors (U/ml) }\end{array}$ & $\begin{array}{l}\geq 500 \\
<500\end{array}$ & $\begin{array}{l}46(50.00) \\
13(48.15)\end{array}$ & $\begin{array}{l}46(50.00) \\
14(51.85)\end{array}$ & 0.866 \\
\hline Recurrent type & $\begin{array}{l}\text { Carboplatin-sensitive } \\
\text { Carboplatin-resistant }\end{array}$ & $\begin{array}{l}37(58.73) \\
22(39.29)\end{array}$ & $\begin{array}{l}26(41.27) \\
34(60.71)\end{array}$ & 0.034 \\
\hline FIGO stage & $\begin{array}{l}\text { III } \\
\text { IV }\end{array}$ & $\begin{array}{l}45(48.91) \\
14(51.85)\end{array}$ & $\begin{array}{l}47(51.09) \\
13(48.15)\end{array}$ & 0.788 \\
\hline Tumor grade & $\begin{array}{c}\mathrm{G} 1 / \mathrm{G} 2 \\
\mathrm{G} 3\end{array}$ & $\begin{array}{r}8(47.06) \\
50(49.02)\end{array}$ & $\begin{array}{r}9(52.94) \\
52(50.98)\end{array}$ & 0.881 \\
\hline
\end{tabular}

Values in bold indicate statistical significance $(\mathrm{P}<0.05)$. USP39, ubiquitin-specific protease 39.
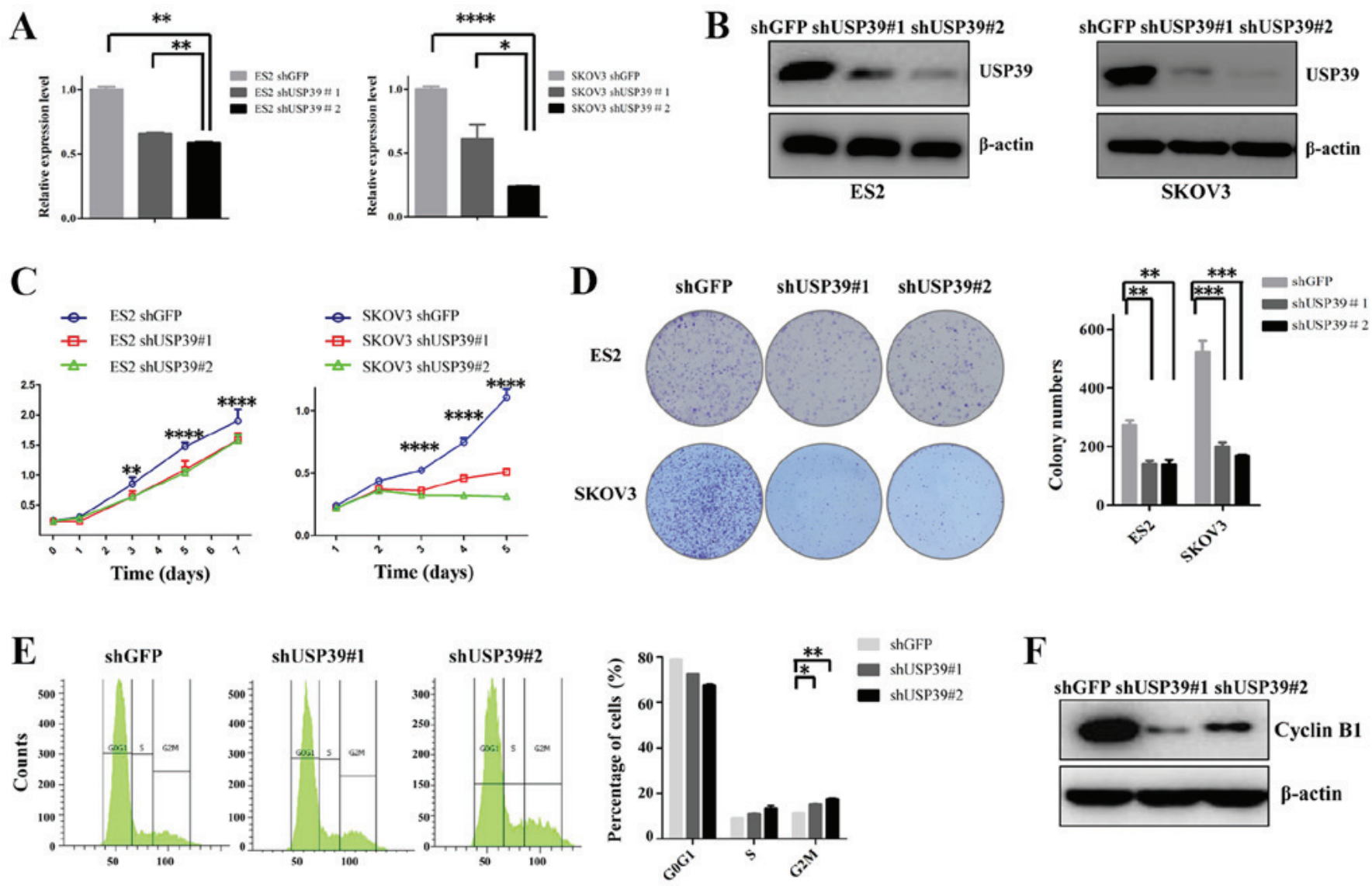

Figure 2. Effect of USP39 knockdown on the growth of ES2 and SKOV3 cell lines. (A) Reverse transcription-quantitative polymerase chain reaction analysis of the messenger RNA levels of USP39 in shGFP and shUSP39s-transduced ES2 and SKOV3 cell lines. $\beta$-actin was used as a reference gene. (B) Identification of knockdown efficiency in shGFP and shUSP39s-transduced ES2 and SKOV3 cell lines by western blot analysis. $\beta$-actin was used as a loading control. (C) The cell proliferative capability was obviously suppressed upon USP39 silencing in ES2 and SKOV3 cells, as measured by Cell Counting kit-8 assay. (D) The number of colonies was significantly decreased following USP39 knockdown in ES2 and SKOV3 cells. (E) USP39 knockdown induced G2/M phase arrest in SKOV3 cells. (F) Western blot analysis of changes in the expression of cell cycle-associated proteins upon knockdown of USP39 in SKOV3 cells. Data represent the means \pm standard error of the mean of 3 independent experiments. shGFP, cells infected with shGFP; shUSP39, cells infected with USP39 shRNA. ${ }^{*}<0.05$, ${ }^{* *} \mathrm{P}<0.01,{ }^{* * *} \mathrm{P}<0.001,{ }^{* * * *} \mathrm{P}<0.0001$, compared with the shGFP group. USP39, ubiquitin-specific protease 39; sh, small hairpin; GFP, green fluorescent protein. 
A

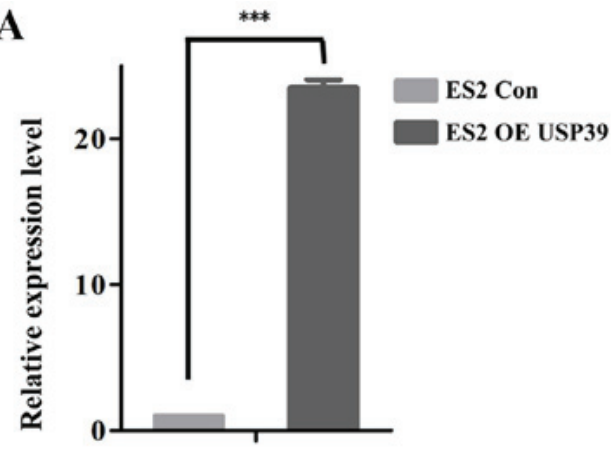

B

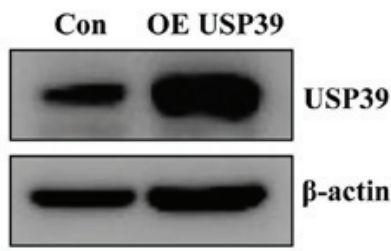

C

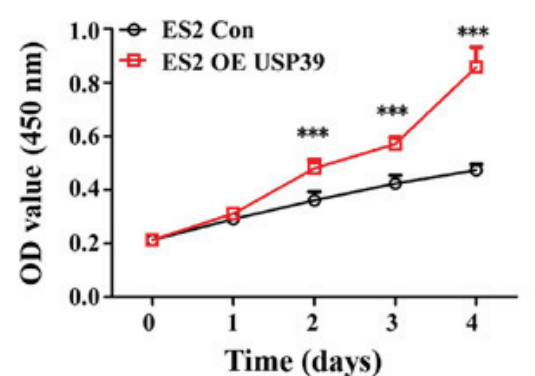

D

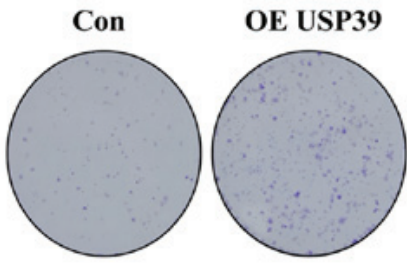

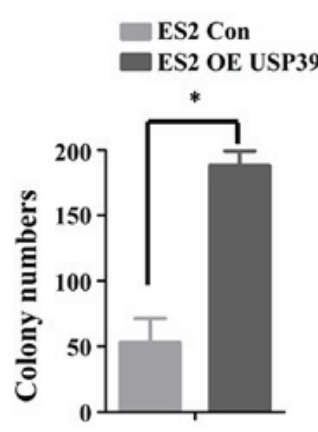

Figure 3. Effects of the overexpression of USP39 on cell proliferation and colony formation of ES2 cells. (A) The expression of USP39 messenger RNA in the USP39-overexpressing ES2 and control cells was examined by reverse transcription-quantitative polymerase chain reaction. (B) Overexpression efficiency of USP39 in ES2 cells was detected by western blot analysis. $\beta$-actin was used as a loading control. (C) Cell proliferation was obviously increased following USP39 overexpression in ES2 cells, as measured by Cell Counting kit-8 assay at $0,24,48,72$ and $96 \mathrm{~h}$. (D) The number of colonies was significantly increased following USP39 overexpression in ES2 cells. ${ }^{*} \mathrm{P}<0.05,{ }^{* * * *} \mathrm{P}<0.001$. Con, ES2 cells infected with pLVX-IRES-Neo; OE USP39, ES2 cells infected with pLVX-IRES-Neo-USP39. USP39, ubiquitin-specific protease 39.

A

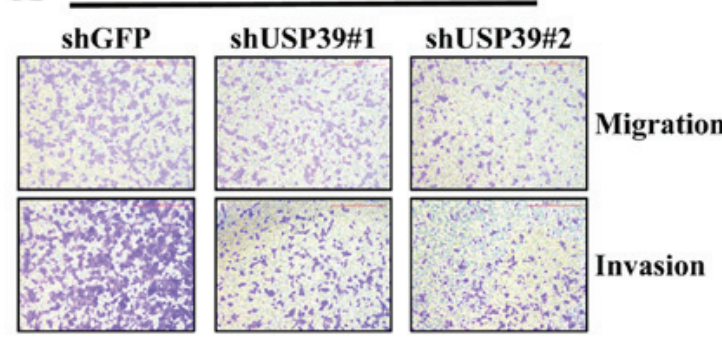

C

C

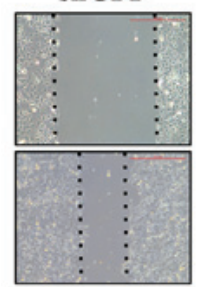

SKOV-3

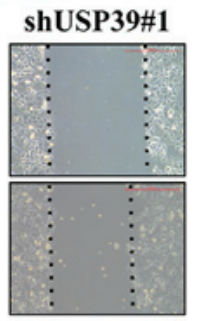

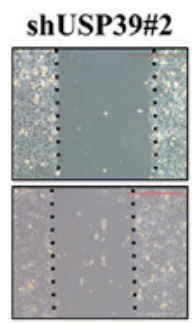

$\mathbf{0 h}$

$48 \mathrm{~h}$

Figure 4. Expression of USP39 in ovarian cancer cells facilitates cell migration and invasion. (A) Representative microscopic views of migratory and invaded cells of sh-green fluorescent protein and shUSP39s-infected ES2 cells were photographed at 100x magnification. (B) A total of 5 fields of the indicated cells were enumerated. The means \pm standard error of the mean from 3 independent experiments performed in duplicate are shown. (C and D) Representative images of wound healing. The knockdown of USP39 suppressed wound healing in SKOV3 cells, whereas the overexpression of USP39 promoted wound healing in ES2 cells. ${ }^{*} \mathrm{P}<0.05,{ }^{* *} \mathrm{P}<0.01$. Images were captured at 0 and $48 \mathrm{~h}$ after wounding. USP39, ubiquitin-specific protease 39 ; sh, small hairpin.

Knockdown of USP39 suppresses ovarian cancer cell migration and invasion. Previous studies have reported that USP39 is a potential target for several types of cancer $(5,6)$. To explore the possibility that USP39 activity may be involved in the
B

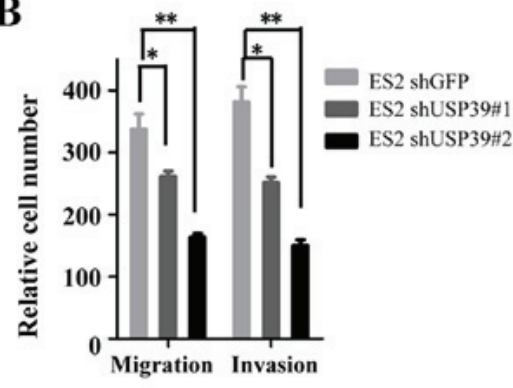

D

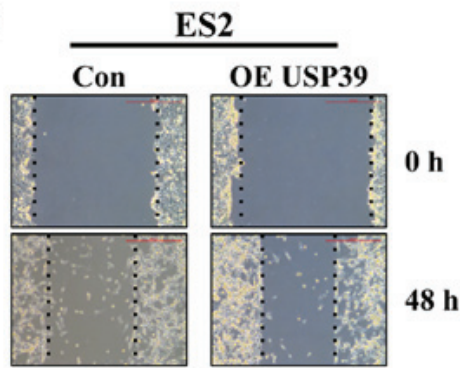

metastatic behaviors of ovarian cancer cells, migration and invasion assays were performed in vitro. USP39 knockdown significantly suppressed the migration and invasion of ES2 cells (Fig. 4A and B). Similarly, the capacity of wound healing was 

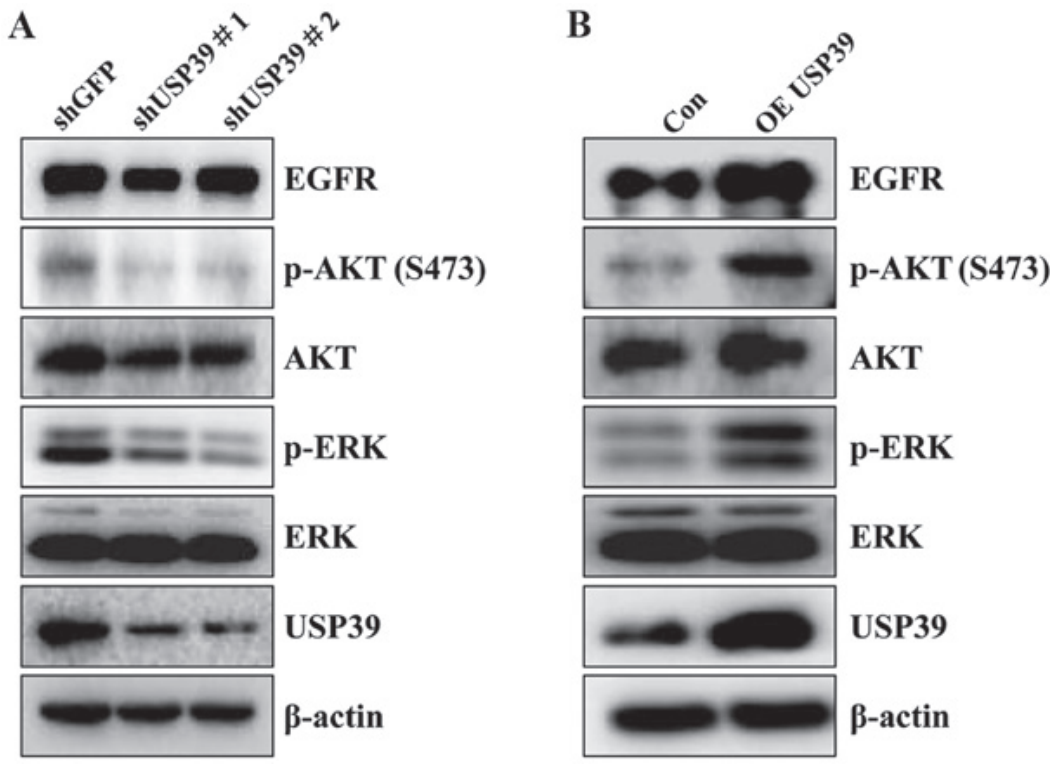

Figure 5. Western blot analysis of ES2 untransfected and transfected cells. (A and B) Western blot analysis of for EGFR, ERK, AKT and their corresponding activated forms in total ES2 cell lysates with knockdown or overexpression of ubiquitin-specific protease 39 . $\beta$-actin served as an internal control for protein loading. USP39, ubiquitin-specific protease 39; EGFR, epidermal growth factor receptor; ERK, extracellular signal-regulated kinase; sh, small hairpin; OE, overexpression.

markedly decreased in the SKOV3 cells in which USP39 was silenced and increased in the USP39-overexpressing ES2 cells compared with their respective control cells (Fig. 4C and D). These results indicate that USP39 plays a potential role in the induction of cell migration and invasion in ovarian cancer.

USP39 is involved in the regulation of AKT and ERK signaling pathways. To ascertain the regulatory mechanisms of action of USP39 as regards the tumorigenesis of ovarian cancer, multiple signaling pathways were analyzed in the ES2 cells upon altering USP39 expression. As shown in Fig. 5, USP39 knockdown markedly decreased the expression of EGFR, phosphorylated (p)-ERK and p-AKT. On the contrary, the expression levels of EGFR, p-ERK and p-AKT were increased upon the overexpression of USP39.

Reduced USP39 levels sensitize ovarian cancer cells to carboplatin treatment. Based on the aforementioned histological and clinical analyses, USP39 may play a role in the regulation of patient's response to carboplatin treatment. Thus, to clarify the possible mechanisms involved in the expression level of USP39 in regulating the apoptosis induced by carboplatin in ovarian cancer cells, flow cytometry was performed and the expression of apoptosis-associated proteins was examined. USP39 deficiency significantly potentiated the apoptotic rate induced by carboplatin in the SKOV3 cells, whereas USP39 overexpression markedly inhibited the apoptotic rate of the ES2 cells (Fig. 6A and C). Consistent with the effects observed on the cell apoptotic rate exerted by USP39, higher levels of cleaved PARP and cleaved caspase-3 were observed in the ES2 cells in which USP39 was silenced than in the control cells (Fig. 6B). By contrast, USP39 overexpression in the SKOV3 and ES2 cells significantly suppressed carboplatin-induced apoptosis, as demonstrated by the marked decrease in cleaved PARP and cleaved caspase-3 levels (Fig. 6D). These data indicate that USP39 plays an important role in mediating the sensitivity of ovarian cancer cells to carboplatin.

USP39 knockdown reverses the promoting effects of USP39 on the chemosensitivity of the ovarian cancer cells to carboplatin, and on migration and invasion. Rescue assays were conducted to prove the function of USP39 in the chemosensitivity of ES2 cells to carboplatin, and its effects on migration and invasion. As shown in Fig. 7A and B, shUSP39\#1 partly attenuated the promoting effects of USP39 on the chemosensitivity of the cells to carboplatin, as evidenced by the increased cell apoptotic rate, and the increased expression of cleaved PARP and cleaved caspase-3 in the shUSP39\#1-transduced cells. Moreover, cell migration and invasion promoted by USP39 was partially recovered by shUSP39\#1 (Fig. 7C). Taken together, these findings demonstrated USP39 promoted the chemoresistance of ovarian cancer to carboplatin, as well as migration and invasion.

Expression level of USP39 influences the chemosensitivity of ES2 and SKOV3 cells to carboplatin in vivo. To further validate the in vitro results, the present study examined the effects of USP39 on the chemosensitivity of ES2 and SKOV3 cells in vivo. Mice were randomly divided into 4 groups ( $n=6 /$ group). The results revealed that the ectopic expression of USP39 significantly increased tumor size. Carboplatin treatment markedly suppressed the growth of ES2 cells in the animal model. The overexpression of USP39 led to a more rapid growth rate with carboplatin treatment (Fig. 8A-C). Mice were randomly divided into 6 groups ( $n=8 /$ group). The results revealed that the knockdown of USP39 reduced tumor burden and carboplatin treatment markedly suppressed the growth of SKOV3 cells in the animal model (Fig. 9). IHC revealed a lower level of cleaved caspase-3 in the USP39-overexpressing cells than in the control cells treated with carboplatin 
A

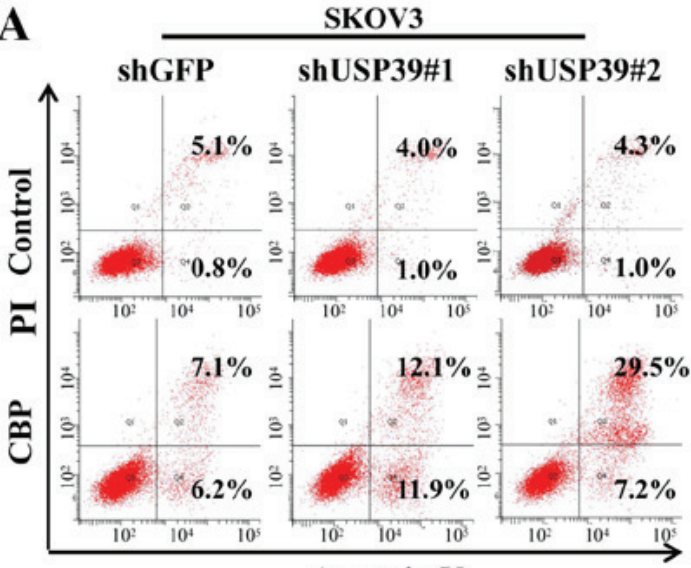

Annexin V

C

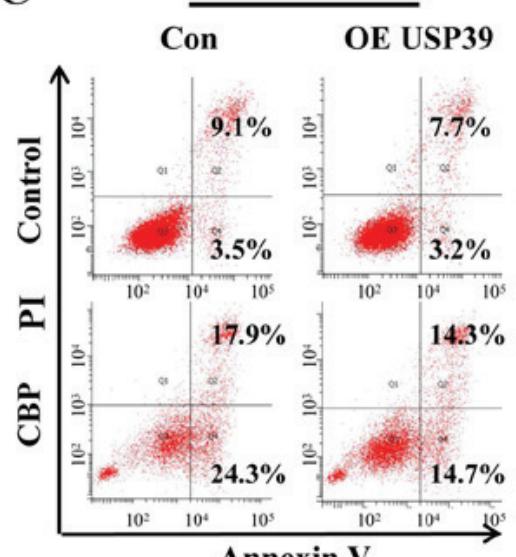

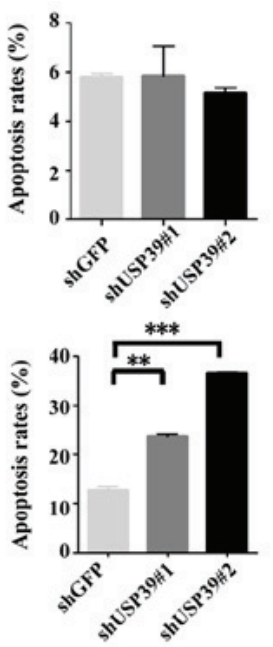

D
B

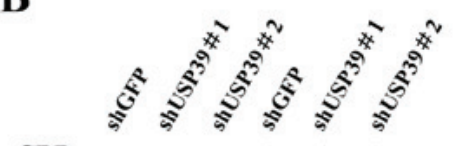

CBP
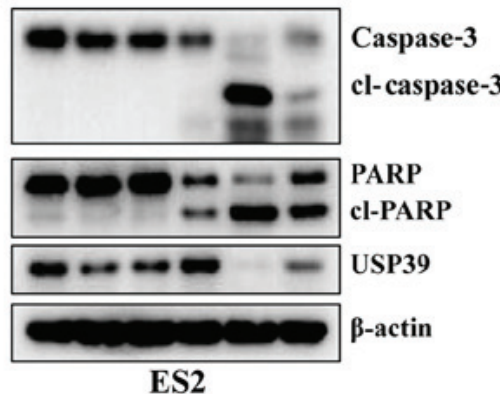

ES2

Annexin V
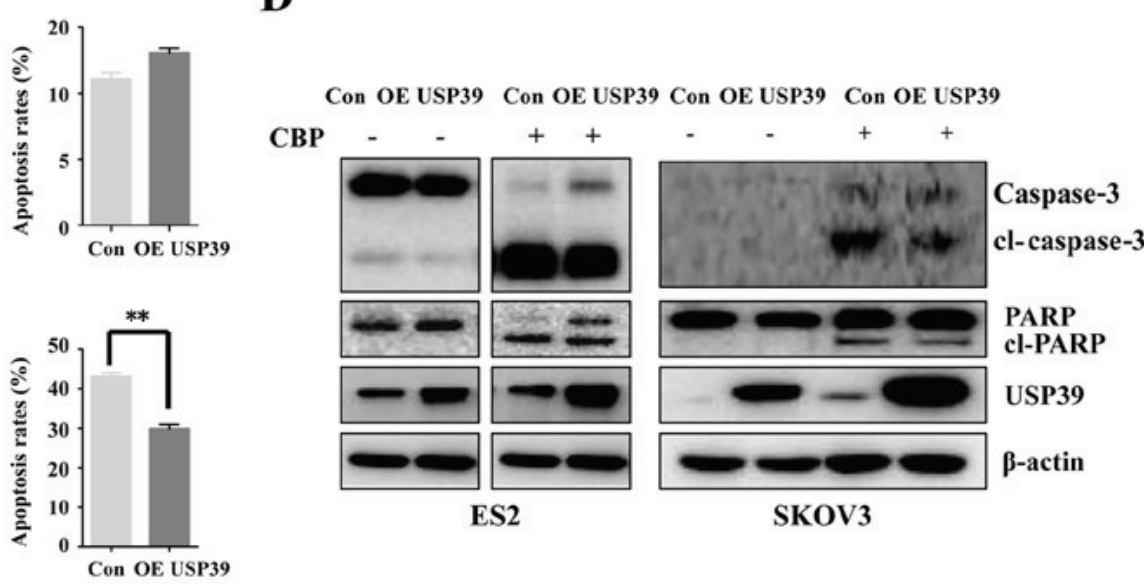

Figure 6. USP39 mediates the sensitivity of carboplatin treatment. (A) The apoptotic rates were analyzed using flow cytometry, which revealed that USP39 knockdown promoted the carboplatin $(450 \mu \mathrm{g} / \mathrm{ml})$-mediated apoptosis of SKOV3 cells. (B) Western blot analysis confirmed that carboplatin (150 $\mu \mathrm{g} / \mathrm{ml})$ treatment promoted apoptosis in ES2 cells, as demonstrated by the marked increase in cleaved PARP and cleaved caspase-3 expression. USP39 knockdown inhibited carboplatin-induced cleaved PARP and caspase-3. (C) The apoptotic rates were analyzed using flow cytometry, which revealed that USP39 overexpression suppressed the carboplatin-mediated apoptosis of ES2 cells. (D) Western blot analysis of apoptosis-associated markers in SKOV3 and ES2 cells treated with carboplatin. ${ }^{* *} \mathrm{P}<0.01,{ }^{* * *} \mathrm{P}<0.001$. USP39, ubiquitin-specific protease 39; PARP, poly-ADP ribose polymerase; sh, small hairpin; OE, overexpression.

(Fig. 8D). These results suggested that USP39 attenuated the chemosensitivity to carboplatin administration. To further investigate the role of USP39 in ovarian cancer metastasis, an experimental murine peritoneal metastasis model was used. The overexpression of USP39 in the ES2 cells promoted the formation of peritoneal metastases (Fig. 8E and F). Taken together, these results demonstrate that USP39 promotes the malignant phenotype of ovarian cancer cells.

\section{Discussion}

As one of the most common gynecological malignancies, ovarian cancer is a major contributor to the high rate of mortality associated with cancer among women due to the absence of methods for early diagnosis of this disease $(16,17)$. Thus far, platinum-based chemotherapy is the most common strategy for ovarian cancer post-operative chemotherapy (18). To the best of our knowledge, therapeutic resistance is one of the major obstacles to the successful treatment of ovarian cancer $(19,20)$. Although remarkable advances have been made in the understanding of the basic mechanisms of cancer over the past decades, the mechanisms of tumor progression and therapy resistance have not yet been entirely determined (21). Thus, there are numerous candidate biomarkers under development to evaluate their efficacy in the treatment of ovarian cancer (22).

The dysregulation of splicing factors has been reported to be associated with cancer development. For example, RNA binding motif protein 4 (RBM4) has been shown to be markedly decreased in patients with cancer $(23,24)$, while serine and arginine rich splicing factor 1 (SRSF1) has been shown to be is highly upregulated in breast and colon cancer $(25,26)$. In this study, the analysis of patients with ovarian cancer that exhibited an overexpression of the spliceosome factor, USP39, suggested that USP39 was involved in carboplatin treatment and USP39 was identified as a potential biomarker of chemotherapeutic resistance in ovarian cancer.

To further confirm our clinical research and explore the biological function of USP39, the effects of USP39 were observed in SKOV3 and ES2 cells. The results helped to uncover the mechanisms of action of USP39 that affect the phenotypes of ovarian cancer cells. Chemotherapeutic treatment resistance could be overcome by the knockdown of USP39. In this study, 
$\mathbf{A}$

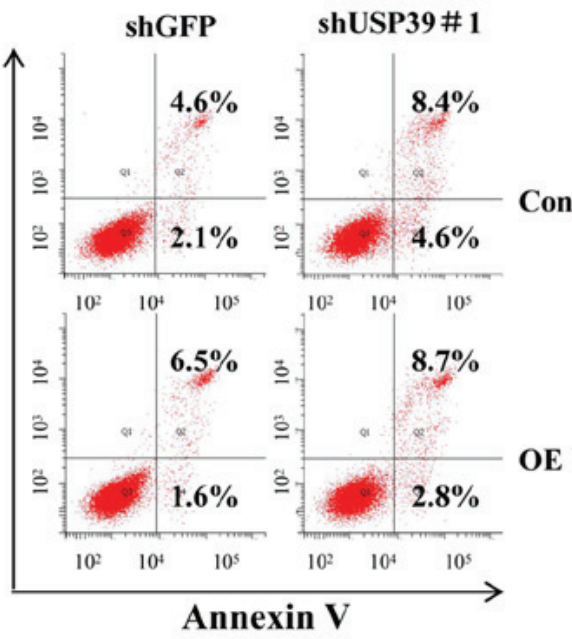

C

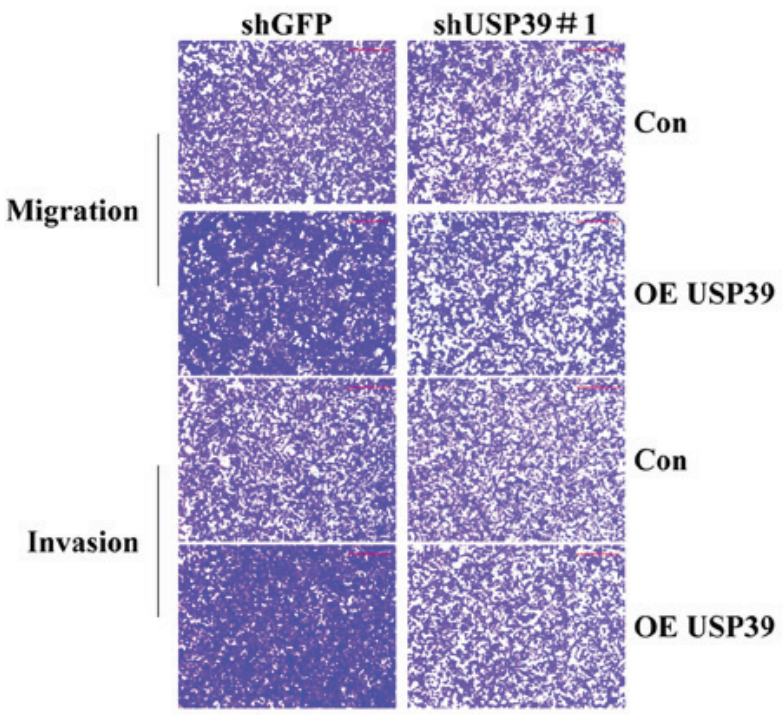

B

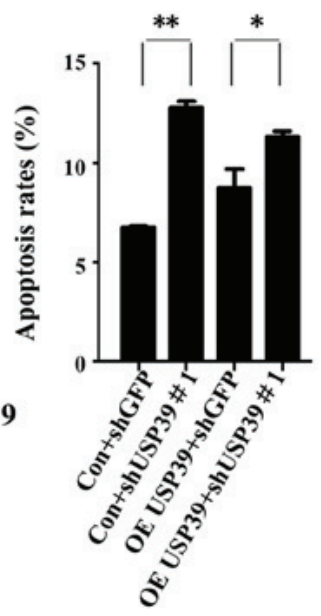

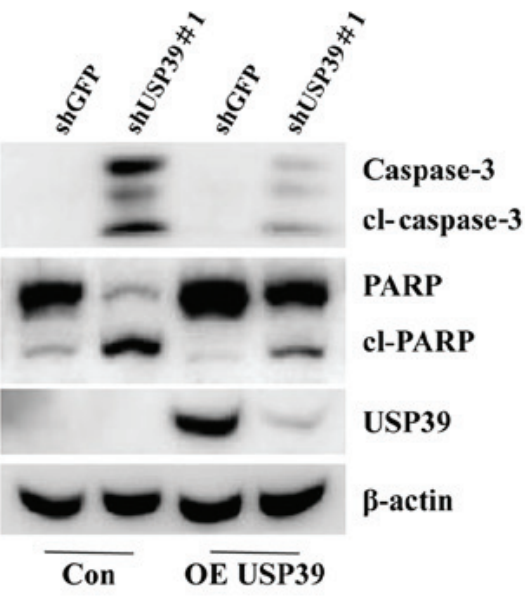

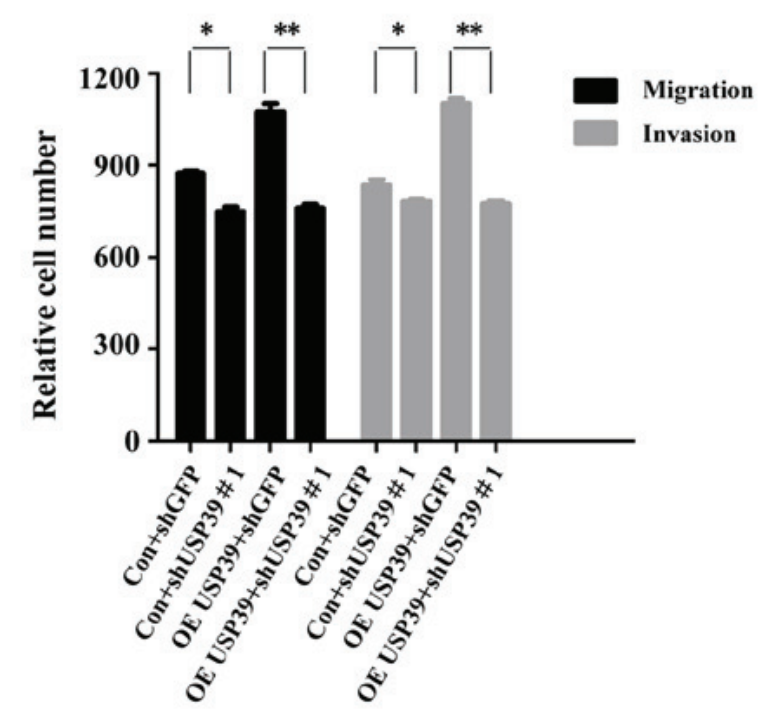

Figure 7. USP39 knockdown reverses the promoting effects of USP39 on cell chemosensitivity to carboplatin, migration and invasion. (A) The apoptotic rates were observed with the Annexin V-FITC-PI Apoptosis Detection kit in ES2 cells treated with carboplatin and co-transfected with pLVX-IRES-Neo/pLVX-IRES-Neo-USP39 and shGFP/shUSP39\#1 vector. (B) Western blot analysis of apoptosis-associated markers in ES2 cells treated with carboplatin. (C) Transwell assay was used to analyze the effects of shUSP39\#1 on USP39-mediated ES2 cell migration and invasion. ${ }^{*} \mathrm{P}<0.05,{ }^{* * *} \mathrm{P}<0.01$, ${ }^{* * * *} \mathrm{P}<0.001$. USP39, ubiquitin-specific protease 39; PARP, poly-ADP ribose polymerase; sh, small hairpin; OE, overexpression.

we demonstrated that the shRNA-mediated downregulation of USP39 resulted in attenuated cell proliferation and colony formation, whereas USP39 overexpression led to an enhanced cell proliferation and colony formation. As stated in a previous study, USP39 participated in the control of cell proliferation, colony formation and apoptosis through PARP cleavage in U2OS cells (27). Previous studies have shown that the induction of $\mathrm{G} 2 / \mathrm{M}$ phase arrest contributes to alterations in the expression of cell cycle regulatory proteins. The knockdown of USP39 was previously identified to arrest progression of the cell cycle at $\mathrm{G} 2 / \mathrm{M}$ phase accompanied by upregulation of p21 (27). In addition, a similar effect was detected in human SMMC-7721 cells, along with the inhibition of Cdc2 activity (28). Similarly, this study demonstrated that the knockdown of USP39 decreased the expression of cyclin $\mathrm{B} 1$, which controls the entrance into mitosis, thereby, accumulating the cell cycle in the quiescent G2/M phase. Although the upregulation of USP39 has been identified to be an oncogenic factor for tumor progression, its molecular mechanisms of action remain to be fully determined. In recent years, the mechanisms contributing to the dysregulation of oncogenes have been investigated (29). It has been reported that the phosphorylation of EGFR and its downstream signaling molecules, such as AKT and ERK can be activated, thereby promoting tumorigenesis and tumor progression (29). In this study, the results revealed that the overexpression of USP39 markedly induced the phosphorylation of EGFR, AKT and ERK. It has been demonstrated that the depletion of USP39 induces U2OS cell apoptosis by PARP cleavage, which is a reliable indicator of apoptosis (27). Notably, this study demonstrated that the silencing of USP39 promoted carboplatin-mediated apoptosis via PARP and caspase-3 cleavage. To validate the function of USP39 in ovarian cancer progression, rescue assays were carried out. The results revealed that the knockdown of USP39 attenuated the promoting effects of USP39 on cell chemosensitivity to carboplatin, migration and invasion, indicating the role of USP39 in ovarian cancer progression. In the in vivo experiments, the overexpression 
A

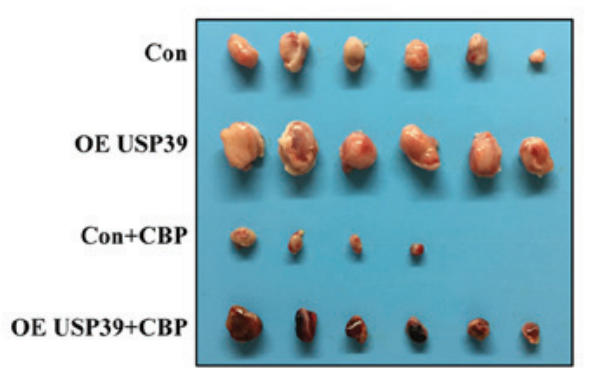

B

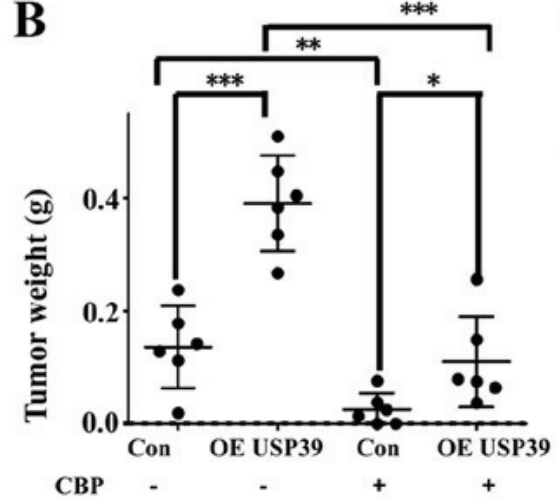

C

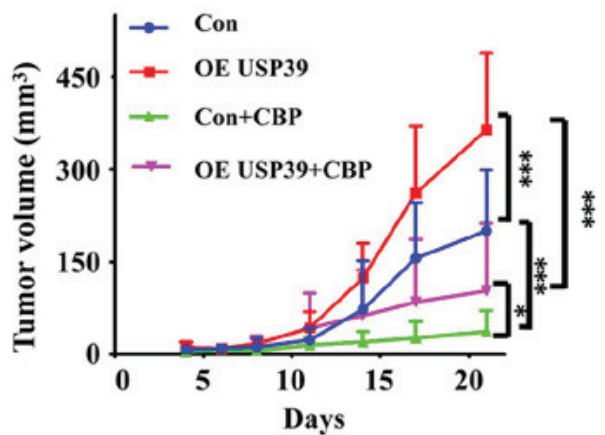

D

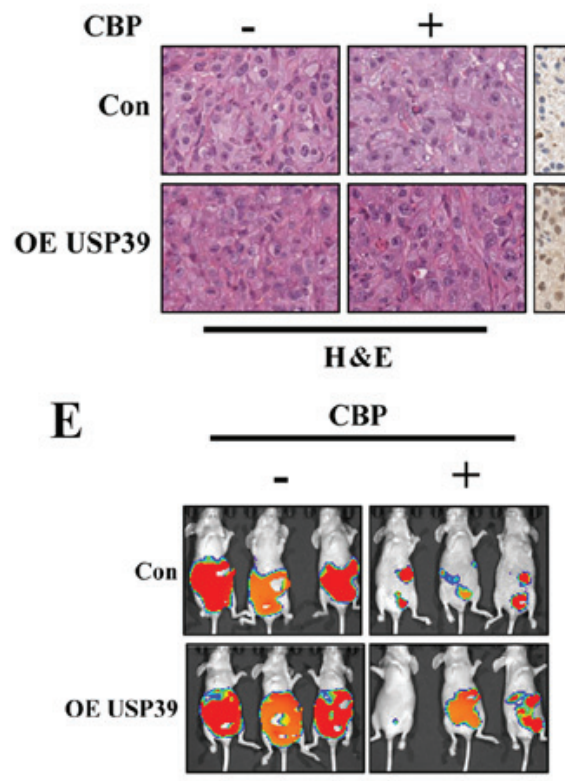

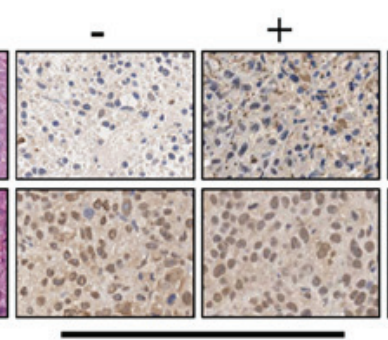

USP39
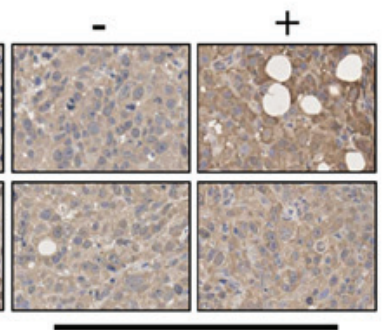

cl-caspase-3

F

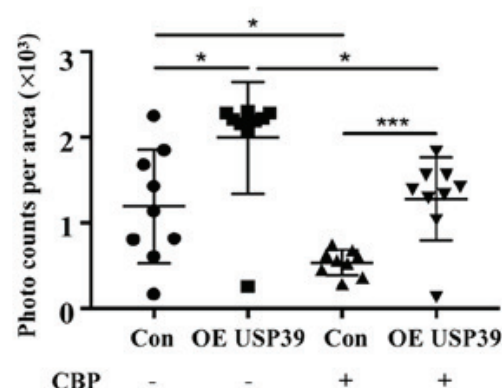

Figure 8. Overexpression of USP39 reduces the sensitivity of ES2 cells to carboplatin and promotes tumor metastasis in vivo. (A) Images of the tumors in the xenograft model. (B) Tumor weight in each group. (C) Tumor volumes in the indicated groups were measured every 3 days. Data are presented as the means \pm standard deviation. (D) The expression of USP39 and cleaved caspase-3 was analyzed by immunohistochemistry in control and USP39-overexpressing ES2 cells. Representative images are shown at $\mathrm{x} 400$ magnification. (E and F) Xenograft models of intraperitoneal metastasis. ${ }^{*} \mathrm{P}<0.05,{ }^{* *} \mathrm{P}<0.01,{ }^{* * *} \mathrm{P}<0.001$. USP39, ubiquitin-specific protease 39; OE, overexpression.

A

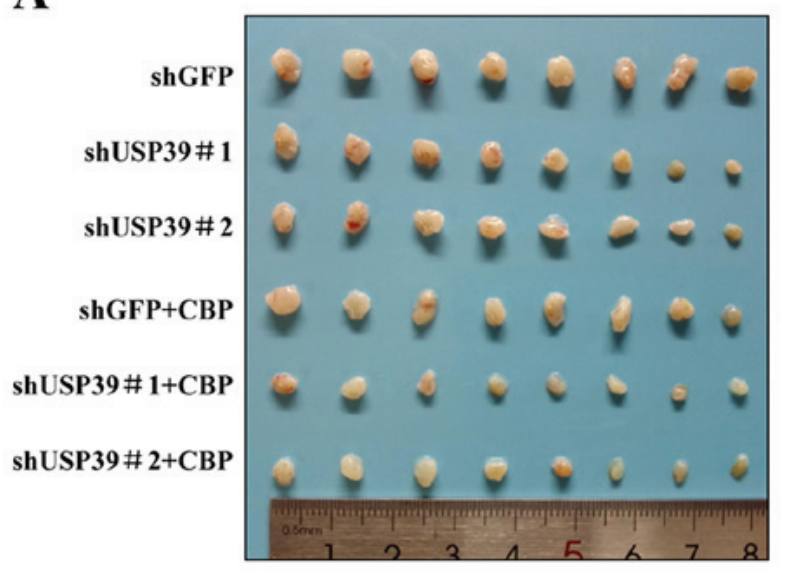

B

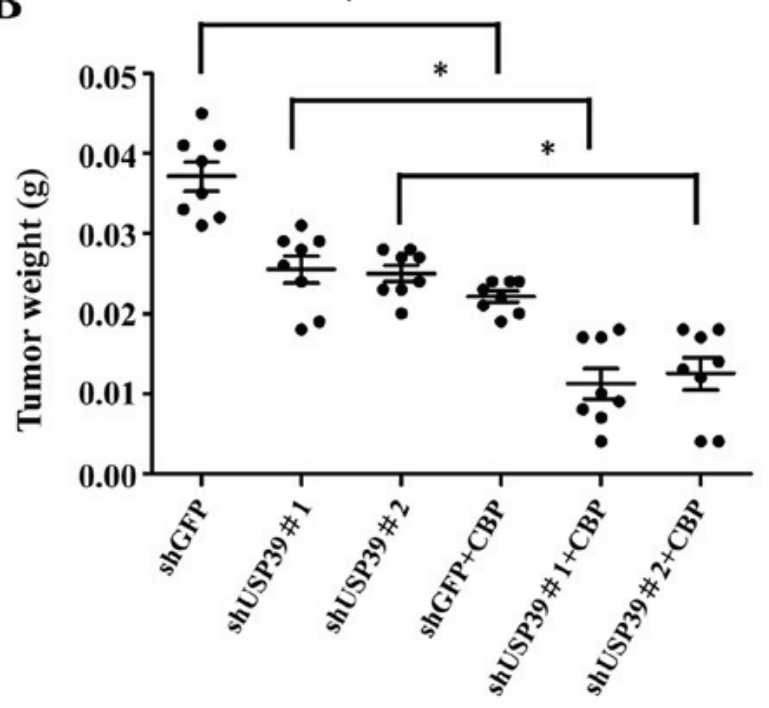

Figure 9. Knockdown of USP39 increases the sensitivity of SKOV3 cells to carboplatin in vivo. (A) Images of the tumors in the xenograft model. (B) Tumor weight in each group. A total of $4 \times 10^{6}$ stably transfected SKOV3 cells were resuspended in $100 \mu 1$ normal saline and injected subcutaneously into the flank of each mouse with a $28 \mathrm{G}$ syringe. At 3 days post-inoculation, carboplatin was administered to the mice by intraperitoneal injection at a dose of $50 \mathrm{mg} / \mathrm{kg}$ every 3 days for 3 consecutive times. ${ }^{*} \mathrm{P}<0.05,{ }^{* *} \mathrm{P}<0.01,{ }^{* * * *} \mathrm{P}<0.001$. USP 39 , ubiquitin-specific protease 39 ; sh, small hairpin. 
of USP39 decreased the chemosensitivity of ES2 cells to carboplatin in subcutaneous xenograft models. By contrast, the knockdown of USP39 reduced the tumor burden. Due to the rapid and early metastasis to the peritoneum in ovarian cancer, it was concluded that USP39 was involved in metastasis in the peritoneal metastasis model. Based on these data, we hypothesized that USP39 promotes ovarian cancer chemoresistance and may thus serve as a therapeutic marker for ovarian cancer.

Taken together, in this study, using in vitro and in vivo approaches, the carcinogenic function of USP39 in ovarian cancer cells was observed for the first time, at least to the best of our knowledge. USP39 knockdown enhanced the sensitivity of ovarian cancer cells to carboplatin. Therefore, USP39 may be used for predicting patients with ovarian cancer who are at a high risk of developing resistance to carboplatin-based chemotherapy. Developing strategies with which to target USP39 may be useful for overcoming therapeutic resistance in patients with ovarian cancer. Several studies have reported a pre-mRNA splicing function for USP39 $(7,10)$. Thus, genes involved in splicing and which are regulated by USP39 should be screened using splicing microarrays in future studies in order to elucidate the mechanisms of action of USP39 in mediating chemoresistance.

\section{Acknowledgements}

Not applicable.

\section{Funding}

This study was supported by the National Natural Science Foundation of China (grant nos. 81650015, 81130043 and 81472452 ), and by the CAMS Initiative for Innovative Medicine (grant no. 2017-I2M-3-004).

\section{Availability of data and materials}

All data generated or analyzed during this study are included in this published article or are available from the corresponding author on reasonable request.

\section{Authors' contributions}

TC conceived the study. LW and TC designed and conducted the experiments. XL, WY and YL conducted data acquisition and data analysis. TC drafted the article and critically revised it for important intellectual content. ZL, HC and ZC contributed to refining the ideas, carrying out additional analyses, processing figures, and finalizing this article. All authors discussed the results, revised the manuscript and agree to be accountable for all aspects of the work. All authors have read and approved the final manuscript.

\section{Ethics approval and consent to participate}

All patients provided informed consent to participate in the study. The study was approved by the ethics committee of the Chinese Academy of Medical Sciences Cancer Hospital (Beijing, China). Animal studies were approved by the
Animal Care and Use Committee of Cancer Hospital, Chinese Academy of Medical Sciences.

\section{Patient consent for publication}

Not applicable

\section{Competing interests}

The authors declare that they have no competing interests.

\section{References}

1. Cheng JQ, Jiang X, Fraser M, Li M, Dan HC, Sun M and Tsang BK: Role of X-linked inhibitor of apoptosis protein in chemoresistance in ovarian cancer: Possible involvement of the phosphoinositide-3 kinase/Akt pathway. Drug Resist Updat 5: 131-146, 2002.

2. Russo A, Czarnecki AA, Dean M, Modi DA, Lantvit DD, Hardy L, Baligod S, Davis DA, Wei JJ and Burdette JE: PTEN loss in the fallopian tube induces hyperplasia and ovarian tumor formation. Oncogene 37: 1976-1990, 2018.

3. van Zyl B, Tang D and Bowden NA: Biomarkers of platinum resistance in ovarian cancer: What can we use to improve treatment. Endocr Relat Cancer 25: R303-R318, 2018.

4. Ricciardelli C, Lokman NA, Ween MP and Oehler MK: Women in cancer thematic review: Ovarian cancer-peritoneal cell interactions promote extracellular matrix processing. Endocr Relat Cancer 23: T155-T168, 2016.

5. Li KY, Zhang J, Jiang LC, Zhang B, Xia CP, Xu K, Chen HY, Yang QZ, Liu SW and Zhu H: Knockdown of USP39 by lentivirus-mediated RNA interference suppresses the growth of oral squamous cell carcinoma. Cancer Biomark 16: 137-144, 2016.

6. Zhao Y, Zhang B, Lei Y, Sun J, Zhang Y, Yang S and Zhang X: Knockdown of USP39 induces cell cycle arrest and apoptosis in melanoma. Tumour Biol 37: 13167-13176, 2016.

7. Ríos Y, Melmed S, Lin S and Liu NA: Zebrafish usp39 mutation leads to rb1 mRNA splicing defect and pituitary lineage expansion. PLoS Genet 7: e1001271, 2011

8. Fraile JM,ManchadoE,Lujambio A,Quesada V,Campos-Iglesias D, Webb TR, Lowe SW, López-Otín C and Freije JM: USP39 Deubiquitinase Is Essential for KRAS Oncogene-driven Cancer. J Biol Chem 292: 4164-4175, 2017.

9. An Y, Yang S, Guo K, Ma B and Wang Y: Reduced USP39 expression inhibits malignant proliferation of medullary thyroid carcinoma in vitro. World J Surg Oncol 13: 255, 2015.

10. Huang Y, Pan XW, Li L, Chen L, Liu X, Lu JL, Zhu XM, Huang H, Yang QW, Ye JQ, et al: Overexpression of USP39 predicts poor prognosis and promotes tumorigenesis of prostate cancer via promoting EGFR mRNA maturation and transcription elongation. Oncotarget 7: 22016-22030, 2016.

11. Wang H, Ji X, Liu X, Yao R, Chi J, Liu S, Wang Y, Cao W and Zhou Q: Lentivirus-mediated inhibition of USP39 suppresses the growth of breast cancer cells in vitro. Oncol Rep 30: 2871-2877, 2013.

12. Li Y, Kong Y, Zhou Z, Chen H, Wang Z, Hsieh YC, Zhao D, Zhi X, Huang J, Zhang J, et al: The HECTD3 E3 ubiquitin ligase facilitates cancer cell survival by promoting K63-linked polyubiquitination of caspase-8. Cell Death Dis 4: e935, 2013.

13. Shu T, Li Y, Wu X, Li B and Liu Z: Down-regulation of HECTD3 by HER2 inhibition makes serous ovarian cancer cells sensitive to platinum treatment. Cancer Lett 411: 65-73, 2017.

14. Livak KJ and Schmittgen TD: Analysis of relative gene expression data using real-time quantitative PCR and the $2(-\Delta \Delta \mathrm{C}(\mathrm{T}))$ Method. Methods 25: 402-408, 2001.

15. Wu X, Luo Q, Zhao P, Chang W, Wang Y, Shu T, Ding F, Li B and Liu Z: MGMT-activated DUB3 stabilizes MCL1 and drives chemoresistance in ovarian cancer. Proc Natl Acad Sci USA 116: 2961-2966, 2019.

16. Ueno M, Shiomi T, Mochizuki S, Chijiiwa M, Shimoda M, Kanai Y, Kataoka F, Hirasawa A, Susumu N, Aoki D, et al: ADAM9 is over-expressed in human ovarian clear cell carcinomas and suppresses cisplatin-induced cell death. Cancer Sci 109: 471-482, 2018. 
17. Xu Y, Miao C, Jin C, Qiu C, Li Y, Sun X, Gao M, Lu N and Kong B: SUSD2 promotes cancer metastasis and confers cisplatin resistance in high grade serous ovarian cancer. Exp Cell Res 363: 160-170, 2018.

18. Longoria TC and Tewari KS: Pharmacokinetic drug evaluation of niraparib for the treatment of ovarian cancer. Expert Opin Drug Metab Toxicol 14: 543-550, 2018.

19. Ricciardelli C, Lokman NA, Sabit I, Gunasegaran K, Bonner WM, Pyragius CE, Macpherson AM and Oehler MK Novel ex vivo ovarian cancer tissue explant assay for prediction of chemosensitivity and response to novel therapeutics. Cancer Lett 421: 51-58, 2018.

20. Wei W, Giulia F, Luffer S, Kumar R, Wu B, Tavallai M, Bekele RT and Birrer MJ: How can molecular abnormalities influence our clinical approach. Ann Oncol 28: viii16-viii24, 2017.

21. Zhang P, Zhang P, Shi B, Zhou M, Jiang H, Zhang H, Pan X, Gao H, Sun H and Li Z: Galectin-1 overexpression promotes progression and chemoresistance to cisplatin in epithelial ovarian cancer. Cell Death Dis 5: e991, 2014.

22. Birkbak NJ, Li Y, Pathania S, Greene-Colozzi A, Dreze M, Bowman-Colin C, Sztupinszki Z, Krzystanek M, Diossy M, Tung N, et al: Overexpression of BLM promotes DNA damage and increased sensitivity to platinum salts in triple-negative breast and serous ovarian cancers. Ann Oncol 29: 903-909, 2018.

23. Wang Y, Chen D, Qian H, Tsai YS, Shao S, Liu Q, Dominguez D and Wang Z: The splicing factor RBM4 controls apoptosis, proliferation, and migration to suppress tumor progression. Cancer Cell 26: 374-389, 2014.
24. No authors listed: RBM4-regulated alternative splicing suppresses tumorigenesis. Cancer Discov 4: 1253, 2014.

25. Chen L, Luo C, Shen L, Liu Y, Wang Q, Zhang C, Guo R, Zhang Y, Xie Z, Wei N, et al: SRSF1 Prevents DNA Damage and Promotes Tumorigenesis through Regulation of DBF4B Pre-mRNA Splicing. Cell Rep 21: 3406-3413, 2017.

26. Anczuków O, Akerman M, Cléry A, Wu J, Shen C, Shirole NH, Raimer A, Sun S, Jensen MA, Hua Y, et al: SRSF1-regulated alternative splicing in breast cancer. Mol Cell 60: 105-117, 2015.

27. Gan Z, Han K, Lin S, Hu H, Shen Z and Min D: Knockdown of ubiquitin-specific peptidase 39 inhibited the growth of osteosarcoma cells and induced apoptosis in vitro. Biol Res 50: 15, 2017.

28. Pan Z, Pan H, Zhang J, Yang Y, Liu H, Yang Y, Huang G, Ni J, Huang $J$ and Zhou W: Lentivirus mediated silencing of ubiquitin specific peptidase 39 inhibits cell proliferation of human hepatocellular carcinoma cells in vitro. Biol Res 48: 18, 2015.

29. Tuan Anh HL, Tran PT, Thao DT, Trang DT, Dang NH, Van Cuong P, Kiem PV, Minh CV and Lee JH: Degalactotigonin, a steroidal glycoside from solanum nigrum, induces apoptosis and cell cycle arrest via inhibiting the EGFR signaling pathways in pancreatic cancer cells. BioMed Res Int 2018: 3120972, 2018. 